\title{
$\mathrm{ICONO} 14$
}

\section{Niños y jóvenes ante los dispositivos: nuevos fenómenos comunicativos y nuevos creadores de producto}

\section{Children and young people in front of the}

devices: new communication phenomena and new product creators

\section{Crianças e jovens diante dos dispositivos: novos fenômenos de comunicação e criadores de novos produtos}

Esther Martínez Pastor ${ }^{1}$ (D)

Ricardo Vizcaíno Pérez ${ }^{2}$ (D)

Ismael López Medel ${ }^{3}$ iD

${ }^{1}$ Profesora Titular, Facultad de Ciencias de la Comunicación (Universidad Rey Juan Carlos), España

${ }^{2}$ Profesor Contratado Doctor, Facultad de Ciencias de la Comunicación (Universidad Rey Juan Carlos), España

${ }^{3}$ Director of the Public Relations program, Department of Communication Studies - College of The Arts (Azusa Pacific University), Estados Unidos

Recibido: 17/12/2021; Publicado: 01/01/2022

Para citar este artículo: Martínez Pastor, E., Vizcaíno Pérez, R. y López Medel, I. (2022). Niños y jóvenes ante los dispositivos: nuevos fenómenos comunicativos y nuevos creadores de producto. Icono 14, 20(1). https://doi.org/10.719 5/ri14.v20i1.1811

Resumen 
Los creadores de contenidos en las diferentes plataformas (como YouTube, Instagram o TikTok) son cada vez son más jóvenes. Los niños y adolescentes son protagonistas de este fenómeno tanto en la creación como en el consumo de los mismos. Los medios tradicionales se han relegado a un segundo plano dejando un claro protagonismo a las plataformas de intercambios de contenido (videos, fotografías, etc.). A través de estos últimos generan mensajes hacia sus iguales con los mismos lenguajes, códigos, preferencias y gustos. Esto promueve un gran interés de las marcas comerciales para estar presentes en este entorno digital. Estas son las cuestiones en las que ahonda este monográfico, donde sus textos nos recuerdan viejos fantasmas de la educación en medios, nos muestran los principales rasgos de los contenidos creados por niños como influencers, las posibles razones por los que algunos influencers consiguen agradar a sus seguidores o la actitud crítica de la audiencia frente a la relación menores-publicidad. El fenómeno fan y su canalización desde las series de ficción a las redes sociales completan el panorama del monográfico.

Palabras clave: Menores; niños; jóvenes; consumidor; producto; influencer; redes sociales; dispositivos móviles

\begin{abstract}
Content creators across platforms such as YouTube, Instagram, or TikTok are increasingly younger. Children and adolescents are the protagonists of this phenomenon both in content creation and consumption. Traditional media have been relegated to a secondary role, opening the door to platforms to exchange content (videos, images). In such platforms, young creators generate messages to their peers using their same language, codes, preferences, and taste. This, in turn, sparks interest in commercial brands to be present in this digital realm. Such are the questions that this special issue investigates. Its articles remind us of the old ghosts of media literacy, showing us the main features of the content created by children as influencers, the potential reasons some influencers manage to connect with their follower base, or the critical audience attitude towards advertising targeting minors. Lastly, this special issue covers the fan phenomenon and its transposition from fiction television to social networks.
\end{abstract}

Keywords: Minors; kids; young people; consumer; producer; influencer; social networks; mobile devices

\title{
Resumo
}

Os criadores de conteúdos em diferentes plataformas (tais como YouTube, Instagram ou TikTok) são cada vez mais jovens. As crianças e os adolescentes são protagonistas deste fenómeno, quer na criação quer no consumo de conteúdos. Os meios tradicionais foram postos em segundo plano, deixando num papel protagonista às plataformas de partilha de conteúdos (vídeos, fotografias, etc.). Através destas últimos, os jovens produzem mensagens para os seus colegas com as mesmas linguagens, códigos, preferências e gostos. Isto promove um grande interesse das marcas comerciais de estarem presentes neste ambiente digital. Estas são as questões que esta monografia explora em profundidade, onde os seus textos lembram-nos velhos fantasmas da educação mediática, apresentam-nos as principais características do conteúdo criado pelas crianças como influenciadores, abrangem as possíveis razões pelas quais alguns influenciadores conseguem agradar aos seus seguidores ou a atitude crítica do público face à relação entre os menores e a publicidade. O fenómeno fã e a sua canalização desde séries de ficção até as redes sociais completam o panorama da monografia. 
Palavras-chave: Menores; crianças; jovens; consumidores; produtos; influenciadores; redes sociais; dispositivos móveis

\section{Presentación}

Como sucede cíclicamente, existe una preocupación social latente ante la relación niños-medios de comunicación. En concreto, en las últimas décadas, la relación niñosdispositivos móviles tal vez sea la que mejor describa esta preocupación. El mundo académico también se ha hecho eco de estas inquietudes y existen numerosos estudios y estadísticas al respecto. Así, estudios de consumo muestran cómo (en determinados contextos) casi el 100\% de los niños entre 5-15 años tienen acceso online (Ofcom, 2021). Se puede afirmar, por tanto, que en un entorno desarrollado el acceso de los niños a las pantallas está normalizado. Sin embargo, esta normalización no ha supuesto dejar una preocupación: la de la larga trayectoria en la que se sitúan los estudios de riesgos de los menores tanto desde el ámbito comunicativo (Garmendia, Martínez y Garitaonandia, 2021; Sic-Spain, 2020; García, López y Montes, 2020), como del consumo, que entre menores y jóvenes es cada vez mayor (Orús, 2021; IAB-PWC, 2019; Holloway; Green; Livingstone, 2013). A ello se unen las preocupaciones desde el punto médico-psicológico (Stoilova et al., 2021; Lee, Kim y Lee, 2019). Y si el enfoque lo ponemos en las plataformas de intercambio de videos los estudios se están dando también desde dos grandes perspectivas: la de la protección de los niños como creadores de contenidos y la de los menores como consumidores de estas plataformas (McLaughlin, 2013; Staksrud, 2013; Lievens, 2010; Lievens, Dumortier y Ryan, 2006).

Pero es la interacción de estos diversos agentes (consumidores, productores y anunciantes) en lo que ahora es preciso profundizar. Así, los efectos (positivos o negativos) ante la presencia de la publicidad en redes sociales siguen siendo un tema por concretar. Las marcas entraron de lleno, en general sin plantearse ni determinadas cuestiones éticas ni legales (cuando estaba recogido claramente en la regulación general de la publicidad, de la competencia desleal, así como, en numerosos códigos de autorregulación de distintos sectores, véase el caso de la publicidad encubierta recogido expresamente tanto en el artículo 9 de la Ley 34/1988, de 11 de noviembre, General de Publicidad, como en art. 26 de la Ley 3/1991, de 10 de enero, de Competencia Desleal) ni determinadas consecuencias negativas que pudieran darse (Carlson et al., 2021): es también un viejo error ya tratado en otros contextos, como el de la publicidad a través del teléfono móvil (García, Niño y Gómez, 2011).

El acceso universal a los dispositivos, del que hablábamos al principio, unido a la ausencia explícita de unas normas legales adaptadas (o más bien el querer mirar hacia otro lado) ha llevado a que los jóvenes, e incluso los menores (alentados por sus padres) se conviertan en productores profesionales de contenidos, ya que en numerosas ocasiones los padres son 
protagonistas con sus hijos de los vídeos (Martínez-Pastor, Blanco-Ruiz y Vízcaíno-Pérez, 2021 y Evans, 2020) y son quienes cobran las ganancias de los menores por la publicidad que aparece (Nieto Moreno y Sosa Troya, 2019). La necesidad de una regulación que concilie los intereses de productores, publicistas y consumidores es patente, y entidades europeas y norteamericanas se han hecho eco de este fenómeno para intentar regular este incipiente mercado online y proteger a los niños. Tal es el caso de las directivas de la UE sobre publicidad, algunos informes de la European Advertising Standards Alliance (EASA) y la Advertising Standards Authority (ASA) en el Reino Unido, así como los informes de la $I A B$ en España (IAB, 2015). En Estados Unidos se han elaborado diferentes guías al respecto por parte de la Federal Trade Commission (FTC) como "Endorsement Guides: What people are askin" (FTC, 2015) o "Advertising Guidance note on Child Brand Ambassadors" (ASA, 2019), entre otros. Afortunadamente de las recomendaciones (Martínez-Pastor et al., 2016) se está transitando a la acción.

El monográfico de la Revista Icono 14, "Niños y jóvenes ante los dispositivos: nuevos fenómenos comunicativos y nuevos creadores de producto", muestra, a través de sus seis textos seleccionados, una visión de algunas de las principales tendencias sobre el tema. El texto que abre el monográfico, "Pantallas y dispositivos móviles. Una necesaria educación para la comunicación de la infancia", de Agustín García Matilla, nos recuerda algunas realidades actuales que ya eran problema hace al menos dos décadas: desde el concepto tan aparentemente actual de "nativo digital" (acuñado por Prensky a principios de siglo) a la aparente ruptura entre competencia digital y pensamiento crítico. El autor nos muestra la necesidad de huir de los apriorismos y de la "certeza de las evidencias" y a escuchar a los protagonistas de la comunicación en este monográfico: los niños y los jóvenes; una escucha, nos recuerda, que no debe pasar solo por los datos, sino por la escucha activa. Así es posible superar el qué y cuánto se consume al por qué y qué sienten al hacerlo ("la subjetividad de la propia autopercepción"). También plantea un anciano problema: el de la educomunicación como parte necesaria del entramado educativo y como misión de servicio público.

El primer trabajo, titulado "Los niños como creadores de contenido en YouTube e Instagram. Análisis de los formatos empleados, la presencia de padres y marcas comerciales", de Erika Fernández Gómez, Jessica Fernández Vázquez y Beatriz Feijóo Fernández, se centra en un tipo de consumidor de redes sociales especifico, que los autores denominan kidfluencers influencers menores de 15 años) y estudia cómo estos jóvenes creadores generan contenido para YouTube e Instagram. En especial, el artículo se centra en el branded content, el contenido generado para marcas a través de YouTube Kids e Instagram. El éxito de estos jóvenes influencers se debe a su gran capacidad para captar la cultura, el lenguaje y el contenido que su audiencia infantil desea ver en redes sociales. En su investigación, los autores analizan y compara el material videográfico de los doce kidfluencers más importantes de España. El resultado es una combinación de guionización de los mensajes de marca con contenido original, fresco, y adaptado a la audiencia infantil. El estudio también presta atención al papel de los padres y las familias en la actividad mediática de los kidfluencers a la hora de generar contenido para las redes, llegando al 
extremo de invitar a los adultos de la casa a convertirse también en creadores de contenido online.

El segundo artículo, titulado "Youtubers: identificación y motivos de agrado de la audiencia" de Oihane Korres Alonso, Iciar Elexpuru Albizuri, presenta un novedoso estudio que analiza la conexión entre los creadores de contenido en redes sociales y sus audiencias. Los autores analizan 52 perfiles de influencers españoles, de los que seleccionan a 10 para realizar un análisis en profundidad. Los autores también realizan un análisis de contenido de 1000 comentarios de seguidores. El análisis de contenido destaca el componente social, en especial el carácter aspiracional del seguidor de un influencer, que ve a esta figura cómo un objetivo a conseguir dada la facilidad de acceso a las redes sociales. El estudio profundiza en la conexión entre los YouTubers y su público, explicando el sentimiento de aprecio y agradecimiento que los usuarios demuestran hacia los generadores de contenido, que se comprueba a través del contenido de los comentarios y en particular del uso de emoticonos y muestras visuales de aprobación y gratitud. Por último, los autores descubren que la conexión entre los YouTubers y su audiencia también genera una identificación personal con el YouTuber del que se aprecia su inteligencia, éxito, carácter emprendido, y en especial su personalidad y sentido del humor.

El tercer artículo profundiza en la relación audiencia-publicidad a través de un estudio de caso: "La aparición de menores en contenidos comerciales y su efecto negativo en la audiencia: el sharenting y la youtuber Verdeliss" (Angeriñe Elorriaga Illera, Sergio Monge Benito, Elena Olabarri Fernández) introduce al lector en el controvertido mundo de los niños como actores o parte del contenido del canal. Los autores recogen preocupaciones en torno a los riesgos de los menores (Livinstone, 2009, 2011; Jiménez et al., 2018) que en el estudio convergen en el denominado sharenting o la exposición pública que los propios padres hacen del menor. A partir de una encuesta a 1336 seguidoras de la youtuber Verdeliss, el texto demuestra que existe una crítica (consciente o inconsciente) sobre dicha presencia que incluso perjudica las acciones comerciales de las marcas. Ello resulta alentador, porque más allá de los posibles límites que legalmente se puedan imponer al uso de los menores (desde la exposición pública de su imagen hasta la preservación de los intereses y derechos del menor, incluso desde una perspectiva laboral), lo que debería primar es una conciencia colectiva (y profesional) de respeto hacia ellos. En todo caso, estos hallazgos pueden ayudar a que las marcas orienten de una forma más adecuada la relación menores-publicidad.

Los seguidores, convertidos en fanáticos, es el objeto de estudio en “La creación de contenidos de ficción juveniles en redes sociales: interacción entre comunidades de fans y plataformas de TV"' (Alba García-Vega y Julián de la Fuente Prieto), también con un estudio de caso. A partir de un análisis multimodal presenta el diseño de la serie SKAM España como un entramado en el que las redes sociales, las plataformas, la ficción de la serie y la vida real de los jóvenes fans se confunden, interactúan y emergen espontáneamente. Los viejos conceptos de significados compartidos que citan los autores (Jenkins et al., 2013) cobran un especial significado en este análisis en el que se puede 
apreciar cómo un producto no sólo se muestra en plataformas diversas, sino que se adapta el producto de formas ingeniosas y sorprendentes, nunca antes vistas. El trabajo muestra cómo las redes sociales son un vehículo adecuado como espacio de un fandom, pero que, además, dicho espacio puede ser reforzado por otras experiencias interactivas satélites o de inmersión en el contenido. El texto también contribuye a aclarar que para el éxito de este fenómeno es necesario adaptar el contenido a las rutinas del público (en este caso adolescente) en cuanto a tiempo y espacio se refiere.

El último artículo, "TikTok y Twitch: nuevos medios y fórmulas para impactar en la Generación Z", de Ainhoa García Rivero, Eva Citlali Martínez Estrella, Gema Bonales Daimiel, pone de manifiesto no sólo la necesidad de adaptar los contenidos comerciales a las diferentes redes sociales, sino que los usos de las mismas por los menores van más allá del mero ocio. El trabajo se articula a través de focusgroup, entrevistas a expertos y una encuesta exploratoria a 420 sujetos (hasta 21 años). El estudio muestra cómo las redes pueden virar hacia otros usos diferentes (incluso "grupos de estudio" del que inicialmente provenían (Twitch-videojuegos). Por otro lado, el mejor recuerdo de marca parece estar relacionado con la modalidad publicitaria elegida (patrocinio de eventos o vídeos), pero también parece haber cierta relación entre el tipo de red y el tipo de modalidad publicitaria (inserción de productos, más habitual en TikTok que en Twitch).

\section{Referencias}

Advertising Standards Authority (ASA) (2019). Advertising Guidance note on Child Brand Ambassadors. Retrieved from: https://cutt.ly/urcqyDK

Carlson, J.R., Hanson, S., Pancras, J., Ross Jr., W.T., \& Rousseau\#Anderson, J. (2021). Social media advertising: How online motivations and congruency influence perceptions of trust. Journal of Consumer Behaviour. doi: https://doi.org/10.1002/cb.1989

Evans, Y.N. (2020). One-sided Social Media Relationships and the Impact of Advertising on Children. Pediatrics, 146(5).

Federal Trade Commission (2015). Endorsement Guides: What people are asking. Retrieved from: ht tps://cutt.ly/krcqnPv

García Guardia, M.L., Niño González, J.I., y Gómez Núñez, P. (2011). Nuevas perspectivas de la publicidad en el teléfono móvil. Relación de los usuarios y la tecnología. Área Abierta, 29, 1-26. http://www.fae.unicamp.br/revista/index.php/etd

García-Jiménez, A., López, M.C.L.D.A., \& Montes-Vozmediano, M. (2020). Características y percepciones sobre el uso de las plataformas de redes sociales y dispositivos tecnológicos por parte de los adolescentes. ZER: Revista de Estudios de Comunicación = Komunikazio Ikasketen Aldizkaria, 25(48). doi: https://doi.org/10.1387/zer.21556

Garmendia, M., Martínez, G. y Garitaonandia, C. (2021). Sharenting, parental mediation and privacy among Spanish children. European Journal of Communication. doi: https://doi.org/10.1177/02 673231211012146

Holloway, D., Green, L., y Livingstone, S. (2013). Zero to eight. Young children and their internet use. London: EU kids online. LSE-The London School of Economics and Political Science. http://epri nts.Ise.ac.uk/52630/1/Zero_to_eight.pdf 
IAB; PWC (2019). Estudio anual de inversión publicitaria en medios digitales 2019. IAB Spain. https:/ /bit.ly/32c64z4

Lievens, E. (2010). Protecting children in the digital era: The use of alternative regulatory instruments. Leiden/Boston MA: Martinus Nijhoff.

Lievens, E., Dumortier, J., \& Ryan, P. (2006). The co-protection of minors in new media: A European approach to co-regulation. Journal of Juvenile Law \& Policy, 10, 97.

Lee, S.Y., Kim, M.S., \& Lee, H.K. (2019). Prevention strategies and interventions for internet use disorders due to addictive behaviors based on an integrative conceptual model. Current Addiction Reports, 6(3), 303-312. doi: https://doi.org/10.1007/s40429-019-00265-z

Martínez-Pastor, E.M., Blanco-Ruiz, M., y Pérez, R.V. (2021). Representaciones familiares en los canales de niños menores creadores de contenidos en YouTube. Un análisis comparado de España, Estados Unidos y Reino Unido. Comunicación y Sociedad, 1-32.

Martínez Pastor, E., Vizcaíno-Laorga, R.I., Ortiz, P., y Riba, A. (2016). Guía Legal de niños influencers. IAB. https://iabspain.es/estudio/guia-legal-ninos-influencers/

Martínez Pastor, E., Vizcaíno-Laorga, R., Ojeda, N., Serrano Maíllo, M.I., y García Maroto, S. (2020). Familias y niños: el negocio de los canales de los niños youtubers. Fundación BBVA. https://bit.I y/3IXKTId

Nieto Moreno, A., y Sosa Troya, M. (2019, 24 de febrero). El polémico negocio de los niños 'youtubers'. El País. https://elpais.com/socie-dad/2019/02/23/actualidad/1550955595_762719 .html

Ofcom (2021). Children and parents: media use and attitudes report 2020/21. https://bit.ly/3p2499f

Orús, A. (10 febrero 2021). Número medio de minutos que la población de 4 a 15 años pasaba en YouTube diariamente en Estados Unidos, Reino Unido y España entre mayo de 2019 y abril de 2020. Statista. https://bit.ly/3F6GWsi

Staksrud, E. (2013). Children in the online world: Risk, regulation, rights. PhD thesis. Aldershot: Ashgate.

Sic-Spain (2020). Actitudes de los niños, niñas y adolescentes hacia el uso seguro de Internet y las Redes Sociales. https://bit.ly/30ydaxs

Stoilova, M., Edwards, C., Kostyrka-Allchorne, K., Livingstone, S., \& Sonuga-Barke, E. (2021). Adolescents' mental health vulnerabilities and the experience and impact of digital technologies: A multimethod pilot study. London: London School of Economics and Political Science. http://ep rints.Ise.ac.uk/112931/ 